\title{
Transposon-directed base-exchange mutagenesis (TDEM): a novel method for multiple-nucleotide substitutions within a target gene
}

\author{
Yun Cheol Kim ${ }^{1}$, Hui Sun Lee ${ }^{3}$, Sukjoon Yoon ${ }^{3}$, and Sherie L. Morrison ${ }^{1,2}$ \\ ${ }^{1}$ Department of Microbiology, Immunology and Molecular Genetics, University of California Los Angeles, Los \\ Angeles, CA, USA, ${ }^{2}$ Molecular Biology Institute, David Geffern School of Medicine, University of California Los \\ Angeles, Los Angeles, CA, USA, and '3Sookmyung Women's University, Department of Biological Sciences, Research \\ Center for Women's Diseases (RCWD), Seoul, Republic of Korea
}

BioTechniques 46:534-542 (June 2009) doi 10.2144/000113152

Supplementary material for this article is available at www.BioTechniques.com/article/113152.

In this report we describe transposon-directed base-exchange mutagenesis (TDEM), an efficient and controllable method for introducing a mutation into a gene. Each round of TDEM can remove up to 11 base pairs from a randomly selected site within the target gene and replace them with any length of DNA of predetermined sequence. Therefore, the number of bases to be deleted and inserted can be independently regulated providing greater versatility than existing methods of transposon-based mutagenesis. Subsequently, multiple rounds of mutagenesis will provide a diverse mutant library that contains multiple mutations throughout the gene. Additionally, we developed a simple framechecking procedure that eliminates nonfunctional mutants containing frameshifts or stop codons. As a proof of principle, we used TDEM to generate mutant lac $Z \alpha$ lacking $\alpha$-complementation activity and recovered active revertants using a second round of TDEM. Furthermore, a single round of TDEM yielded unique, inactive mutants of $c c d B$.

\section{Introduction}

Many different methods of mutagenesis have been developed to alter protein function or activity $(1,2)$. UV $(3-5)$ chemical mutagenesis $(6,7)$, or site-directed mutagenesis $(8)$ have traditionally been used to introduce mutations. Current mutagenesis methods can be categorized into two groups: controlled or random mutagenesis (9).

PCR-based site-directed mutagenesis $(10,11)$ is the method of choice for controlled mutagenesis. Alanine (12-16) or cysteine scanning mutagenesis (17-19) has been widely used to investigate protein function or structure. Additionally, mutator bacterial strains (20-23), gene shuffling (24), and error-prone PCR (epPCR) (25) have been widely used for random mutagenesis. While these methods have been used for many studies, they have several disadvantages (9,26). Site-directed mutagenesis can be costly and labor-intensive, as different mutations require the use of different oligonucleotides, PCR, and cloning. Although random mutagenesis methods can provide mutant libraries using simple procedures, the rate and the site of mutations cannot be controlled. Therefore, there is a risk of high wild-type background and if a gene contains multiple mutations, each mutation must be individually analyzed to identify the amino acid change(s) responsible for the mutant phenotype (24). Although the random insertion and deletion (RID) method was developed to surmount these shortcomings, its use is limited to those who are technically sophisticated (27). As an alternative approach, transposons have recently been used for pentapeptide scanning (28) and linker scanning mutagenesis (29), but these methods are also limited and not appropriate for protein evolution because they only insert extra sequences into the target gene.

With an optimal mutagenesis method, it should be possible to $(i)$ control the number of mutations per round of mutagenesis and the sequence to be inserted or substituted, (ii) have low wild-type background, and (iii) have flexibility so that different types of mutations-including insertions, deletions, or substitutions_can be obtained.
None of the currently available mutagenesis methods satisfies all of these requirements. In order to achieve these characteristics, we developed transposon-directed baseexchange mutagenesis (TDEM), which can substitute up to 11 bases of the target gene with a predetermined sequence of any length into a site selected by the random integration of a transposon (30,31). TDEM introduces a mutation into a single site of the target gene so that the diversity of the mutant library can be easily evaluated. Furthermore, because it can substitute $\geq 3$ bases, TDEM can be used for amino acid scanning mutagenesis using up to 3 consecutive amino acids providing a novel approach for directed evolution.

As a proof of principle, we used TDEM to substitute 3 random bases of the target genes $l a c Z \alpha$ ( $\alpha$ fragment of $\beta$-galactosidase) and $c c d B$ (controller of cell division protein B) with three bases determined by the mutation insert (MI). In both target genes, we showed that $(i)$ one round of TDEM generates a mutation at a single site with no wild-type background, (ii) TDEM can be used to generate a predetermined 


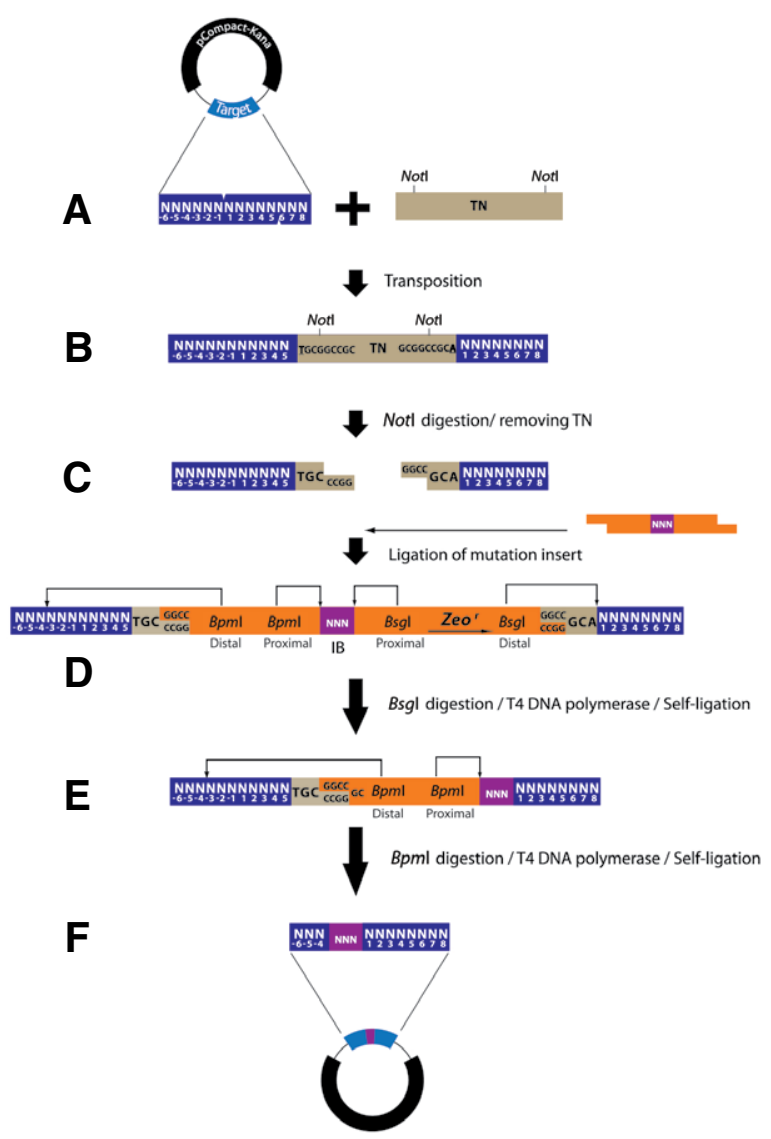

Figure 1. Schematic diagram of TDEM method. The transposon was introduced into the target gene in a plasmid vector (A) and transformed into $E$. coli by electrotransformation. Five bases of the transposon integration site are duplicated and a single $A$ or $T$ added at both ends of the transposon as depicted (B). The plasmid vectors containing the transposon were digested with Notl which cuts at both ends of the transposon (C). The mutation insert (MI) containing the Zeocin resistance gene was cloned into the target gene. The plasmid containing the $\mathrm{MI}$ (D) was digested with Bsgl and selfligated $(E)$. The plasmid was then digested with $B p m$ l that cuts between -4 and -3 base of the left end and at the $5^{\prime}$ end of the NNN sequence that is to be introduced into the gene. The final plasmid $(F)$ has a three base substitution at a random position within the target gene. The NNN sequences used were GCC, CGC, AAC, GAT, TGC, CAG, GAA, GGC, CAT, ATC, CTG, AAA, ATG, TTT, CCA, AGC, ACC, TGG, TAC, or GTG.

3-base substitution at a random site, and (iii) repeated rounds of TDEM accumulate mutations within the gene. Therefore, TDEM exhibited characteristics of both controlled and random mutagenesis. Thus, we propose that the use of TDEM will dramatically facilitate structural studies and directed evolution of proteins.

\section{Materials and methods}

Plasmid constructs and transposons

The pCompact-Kana vector was constructed by ligating an origin of replication with kanamycin resistance gene (Supplementary Figure 1 and Supplementary Table 1). All inserts were cloned into pCompact-Kana, which had been amplified by PCR with primers Kan-up and Ori-lo (Supplementary Table 1) to make a linear plasmid. The detailed methods are in the Supplementary Materials.

\section{Transposition}

For TDEM of lac $Z \alpha$ (150 bp), transposition was carried out using commercial Tn7 transposase (TnsABC* transposase; New England Biolabs, Ipswitch, MA, USA) and Mu-transposase (Finnzymes, Espoo, Finland) according to the manufacturers' protocol. The mixture of $\operatorname{Tn} 7$ and $\mathrm{Mu}$ transposition reactions was used in order to increase the spectrum of possible mutations. For TDEM of $c c d B$ (300 bp), $\mathrm{N}$-terminally deleted MuA prepared in the laboratory was used in the modified buffers that altered site preference (A: 25 $\mathrm{mM}$ Tris-Cl (pH 7.0), $5 \mathrm{mM} \mathrm{MgCl}_{2}, 0.05 \%$ (v/v) Triton X-100, $10 \mathrm{mM} \mathrm{NaCl}$ and $40 \%$ (v/v) glycerol; $\mathrm{B}$ : same as A without $\mathrm{NaCl}$; C: same as A with $15 \%(\mathrm{v} / \mathrm{v}) \mathrm{DMSO}$; and D: same as A with $10 \%$ (v/v) glycerol) (32) and transposition reactions carried out using these buffers were mixed. $T n 7$ was not used for $c c d B$ mutagenesis because it showed batch to batch variation in enzyme activity. Following transposition, the DNA mixture was electrotransformed into Escherichia coli. The transformants were inoculated into LB broth containing $50 \mu \mathrm{g} / \mathrm{mL}$ kanamycin and $3 \mu \mathrm{g} / \mathrm{mL}$ chloramphenicol and cultured overnight at $37^{\circ} \mathrm{C}$ with shaking (Excella E24, New Brunswick Scientific, Edison, $\mathrm{NJ}$, USA). All transposition reactions in this study yielded $>20,000$ transposition events. The plasmid DNA from the pool was harvested and digested with the restriction enzymes that flank the target gene: $\mathrm{NcoI} /$ AgeI (New England Biolabs) for lac $Z \alpha$ or $B s a \mathrm{I}$ (New England Biolabs) for $c c d B$. The target gene carrying the transposon was purified from agarose gels after electrophoresis, ligated back into the parental vector digested with compatible restriction enzymes and electrotransformed again yielding $\sim 1 \times 10^{7}$ colonies. This step elimi- nated plasmids having the transposon in the vector sequence and resulted in a plasmid population with the transposon only in the target gene.

When blunt ends were required, $3 \mu \mathrm{g}$ of purified plasmid DNA containing sticky ends was suspend in $0.5 \mathrm{mMdNTP}$ and $1 \times$ T4 DNA polymerase buffer (New England Biolabs) with 6 units of T4 DNA polymerase (New England Biolabs) and incubated at $12^{\circ} \mathrm{C}$ for $25 \mathrm{~min}$.

\section{Evaluation of transposon \\ integration sites}

The pool of plasmids containing the transposon only in the target gene was harvested and digested with NotI (New England Biolabs), which cuts both ends of $\mathrm{Tn} 7$ (New England Biolabs) and M1-Cam ${ }^{\mathrm{R}}$ (Finnzymes), resulting in a linear plasmid opened at the integration site in the target gene. After agarose gel purification, the linearized plasmid was end-labeled with $\gamma-32 \mathrm{P}$ ATP and then digested with $\mathrm{AgeI}$ (New England Biolabs) (lac $Z \alpha$ ) or PvuII (New England Biolabs) ( $c c d B$ ), which cuts the vector sequence outside of the target gene. The resulting DNA mixture then contains various sized ${ }^{32} \mathrm{P}$-labeled DNA fragments depending on the transposon integration site. The digested DNA fragments were analyzed by $6 \%(\mathrm{w} / \mathrm{v})$ polyacrylamide DNA gel electrophoresis in the presence of $5 \mathrm{M}$ urea.

\section{Mutant Screening}

The $\alpha$-complementation activity of mutant $l a c Z \alpha$ was identified using LB agar containing $50 \mu \mathrm{g} / \mathrm{mL}$ kanamycin, $60 \mu \mathrm{g} / \mathrm{mL}$ $\mathrm{X}$-gal and $1 \mathrm{mM}$ IPTG after overnight incubation at $37^{\circ} \mathrm{C}$. To isolate inactive $c c d B$, the mutated $c c d B$ was harvested from pCompact-Kana-CcdB using $B s a I$ digestion and ligated into the $\mathrm{pFinalScreen}$ vector (Supplementary Figure 1) prepared by removing stuffer DNA with $B$ sal digestion. The ligation mixture was then electro-transformed into Top10 electrocompetent cells (Invitrogen, Carlsbad, CA, USA). Surviving colonies were observed on LB agar plates containing $50 \mu \mathrm{g} / \mathrm{mL}$ kanamycin and $1 \mathrm{mM}$ IPTG after overnight incubation at $37^{\circ} \mathrm{C}$.

Eliminating mutants containing frameshifts or stop codons using pFrameCheck

The mutated $c c d B$ gene was harvested from pCompact-Kana-CcdB by digesting with $B s a \mathrm{I}$ and cloned into $\mathrm{pFrameCheck}$ prepared by digestion with $B s a \mathrm{I}$. The ligated mixture was transformed into DB3.1 electrocompetent cells (Invitrogen) and grown in LB containing $50 \mu \mathrm{g} / \mathrm{mL}$ carbenicillin and 50 $\mu \mathrm{g} / \mathrm{mL}$ kanamycin overnight at $37^{\circ} \mathrm{C}$ with shaking. The plasmid was purified from the 
$E$. coli lysate and the mutant $c c d B$ gene was purified from the agarose gel after digestion with $B s a \mathrm{I}$. The harvested mutant $c c d B$ was cloned into pFinalScreen and screened as described above.

\section{Results}

General procedure of TDEM

The procedure for producing a 3-base substitution using TDEM is outlined in Figure 1 and the Supplementary Materials. The key to the success of the method is the design of the MI. We choose to used $B p m I$ and $B s g \mathrm{I}$ for constructing the MI after testing the activity of several type IIs restriction enzymes including $B p m \mathrm{I}, B p u \mathrm{EI}$, $B s g \mathrm{I}, M m e \mathrm{I}$ and $E c o \mathrm{P} 15 \mathrm{I}$. As shown in Figure $1 \mathrm{D}$, the MI consists of three components, (i) distal BsgI and BpmI sites to remove bases from the target gene, (ii) nucleotides to be inserted into the target gene (incorporating bases, IB) between the proximal $B s g \mathrm{I}$ and $B p m \mathrm{I}$ sites and (iii) a Zeocin resistance gene $\left(z e o^{\mathrm{r}}\right)$. BpmI and BsgI, type IIs restriction endonucleases, cut 16 bp outside their recognition sequence. Therefore, by adjusting the relative position of the distal restriction enzyme sites, the number of bases to be deleted from the target can be altered (Supplementary Figure 2). In Figure 1D, the distal BpmI and BsgI are positioned so that three bases are removed from the target; however, it should be stressed that it is possible to position the enzyme sites so that up to 11 bases are deleted from the target (Supplementary Figure 2). On the other hand, the sequence to be inserted is determined by the IB that is placed between the proximal BpmI and BsgI sites. The IB can be of any length. Thus, the number of bases to be deleted and the length of the DNA to be inserted can be separately determined so that many types of mutant libraries can be generated. Lastly, the $z e o^{\mathrm{r}}$ gene confers resistance to Zeocin so that only plasmids containing the $\mathrm{MI}$ are recovered following selection with Zeocin and kanamycin.

To carry out TDEM, the Mu and/or $\operatorname{Tn} 7$ transposons are first integrated into random sites within the target gene. These transposons contain NotI sites at their ends (Figure 1A). As a result of transposition, the five bases of the integration site are duplicated with the transposon located between the five duplicated bases (33) (Figure 1B). The transposon is then removed by digesting with NotI (Figure 1C). Subsequently, the MI is ligated into the linearized vector (Figure 1D) and transformed into E. coli. The plasmids containing the MI are then digested with $B s g$ I to remove the right hand side of the MI (Figure 1E). After making the plasmid ends blunt with T4 DNA

Table 1. Position and Sequence of Mutations within Inactive $\operatorname{ccdB}$

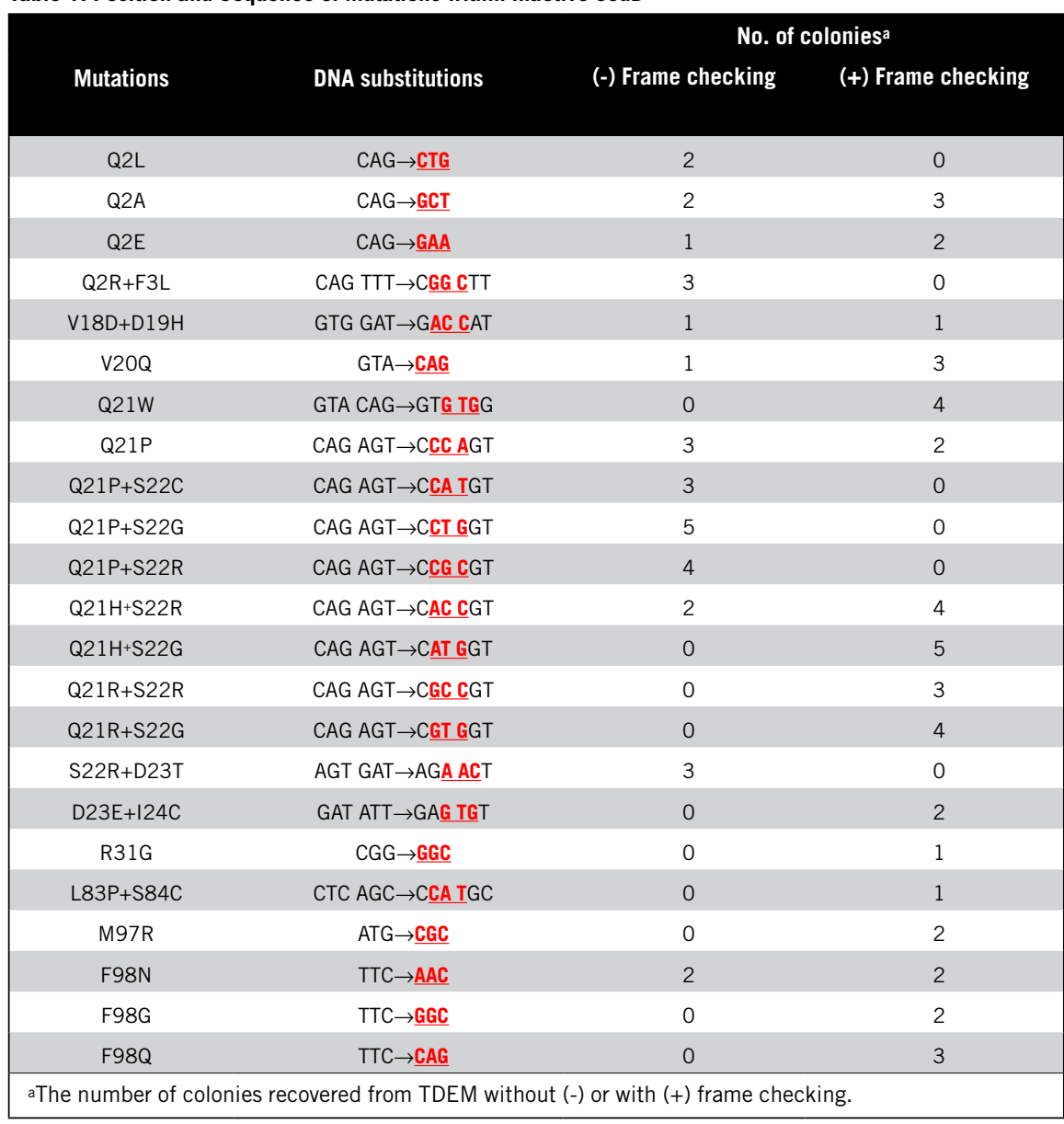

polymerase, the linearized plasmid is selfligated and transformed into $E$. coli. The resulting plasmid is then digested with $B p m I$ to remove the left hand side of MI (Figure 1F). Following treatment with T4 DNA polymerase to create blunt ends, self-ligation and electrotransformation are performed to finally obtain the mutant library containing a substitution of three bases at random positions in the target. The number of colonies recovered after each step was $\sim 1 \times 10^{7}$, and always exceeded the number of colonies recovered in the previous step.

In this study, MIs that substitute three bases were used so that the resulting mutants would have a single amino-acid substitution, or, at most, two. The MI can, of course, generate various mutations depending on the frame and orientation of the MI (Supplementary Figure 3). Thus, it is not possible to completely control the mutations obtained using TDEM. However, this can be an advantage because multiple mutants can be generated using one MI, increasing the diversity of the library. If two codons are affected, it is possible to generate two consecutive amino acid changes, an event that rarely occurs using epPCR (Supple- mentary Materials) or other mutagenesis methods (34).

Frame-checking procedure

In initial studies, we observed that after TDEM, more than half of the clones contained inappropriate deletions or insertions. We speculated that this resulted from inaccurate digestion by the restriction enzymes and/or overdigestion by T4 DNA polymerase. Although the presence of inappropriate mutations is not a problem when an efficient screening system is used to positively select mutants, it results in a high background when isolating loss-of-function mutations. Therefore, the pFrameCheck vector (Figure 2 and Supplementary Figure 1) was constructed so that mutants containing a frameshift or stop codon would be eliminated. In frame-checking, the mutated gene is cloned between the gIII signal sequence of the coat protein of phage $\mathrm{fd}$ (35) and $\beta$-lactamase. Therefore, only when the gIII signal sequence and $\beta$-lactamase are linked in frame through the mutated gene, will the $\beta$-lactamase be transported into the periplasmic space and the clones survive in the presence of ampicillin or carbenicillin (Figure 2). Therefore, mutated target DNA 


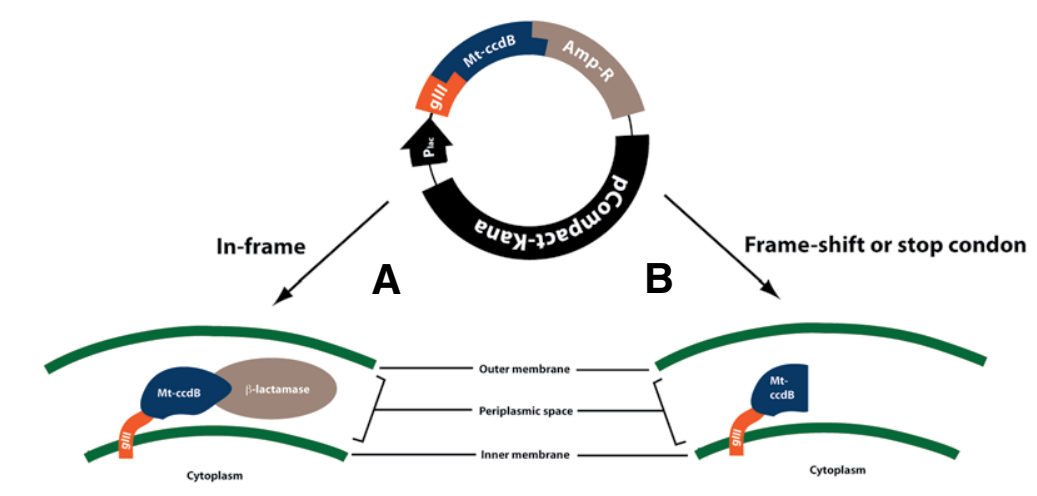

Survival in the presence of ampicillin

Growth inhibition in the presence of ampicillin

Figure 2. Schematic diagram of the frame-checking procedure. The mutant $c c d B$ genes in pCompactKana were purified using Bsal digestion and ligated into pFrameCheck-Kana. The ligation mixture was transformed into $E$. coli and grown on LB agar plates containing kanamycin and carbenicillin overnight. (A) The $\beta$-lactamase gene that is fused to mutant proteins without a frameshift or stop codon will be expressed in the periplasmic space and cells will survive in the presence of carbenicillin. (B) However, when a frameshift or stop codon is present, the $\beta$-lactamase gene fused to the mutant $\mathrm{CcdB}$ will not be expressed in the periplasmic space and the clones will be sensitive to carbenicillin resulting in cell death. Using this procedure, only mutants without a frameshift or stop codon are recovered.

with a frameshift or stop codon will be eliminated resulting in a mutant library in which most, if not all, of the clones contain the desired base substitution.

\section{Mutagenesis of $l a c Z \alpha$ using TDEM}

lac $Z \alpha$ was cloned into pCompact-Kana (Supplementary Figure 1 and Supplementary Table 1). Use of this vector maximizes the possibility of introducing the transposon into the target gene. Plasmids with insertions into an essential part of the vector (origin and kanamycin resistance gene) will not grow. Transposition into lac $Z \alpha$ $(36,37)$ was carried out with Mu and Tn7 resulting in more than 20,000 colonies, and an IB sequence of GCC (GCC-MI) within the MI. Following transposition, transposon integration sites were analyzed as described in the "Materials and methods" section. In the gel, each band represents a site attacked by the transposon ( $\mathrm{Mu}$ and $\operatorname{Tn} 7$ ) with the intensity of the band indicating the frequency of transposition into this site (Figure 3). Although not every base was attacked, when both transposons were used, a relatively even distribution was observed (Figure 3B). The result indicates that the sites mutated by TDEM are located throughout the $l a c Z \alpha$ gene.

lac $Z \alpha$ mutants deficient in $\alpha$-complementation after TDEM with GCC-MI were identified on LB plates containing X-gal. Eight white and 2 pale blue colonies were picked. The 2 pale blue clones contained amino acid substitutions at two different sites (L8A, P32A) (Figure 4 and Supplementary Table 2). Among the 8 white colonies, 2 had the same mutation (N19P) and showed no $\alpha$-complementation activity (Figure 4 and Supplementary Table
2). Among the other 6 white colonies, 2 had one-base deletions and 4 had insertions of 1 or 2 bases, which resulted in frameshifts (Supplementary Table 2). In this study, TDEM was performed without frame-checking and yielded many clones containing unwanted base deletions or insertions. We used the N19P mutant as the target for the next round of mutagenesis.

Second round of mutation of lac $Z \alpha$

The N19P mutant was then used for a second round of TDEM with GCC-MI to generate revertants displaying $\alpha$-complementation activity. Fifteen dark blue colonies were picked and sequenced. All revertants showing the dark blue phenotype had the same mutation, P19A. Two pale blue clones from the second TDEM showed different mutations, P19G and Q24G (Figure 4 and Supplementary Table 2).

In the initial reversion study, the spectrum of revertants recovered was limited because only one MI (GCC-MI) was used. In an attempt to broaden the spectrum of revertants, a mixture of MI containing 20 different IB (MI-mix) was used for TDEM. Subsequently, 20 dark blue clones were picked and sequenced. Although all dark blue revertants were still mutated at P19 (Figure 4 and Supplementary Table 2), these revertants contained a variety of one or two amino acid mutations. The pale blue colonies recovered contained the mutations P19G, Q24G, and T45K+D46N (Figure 4 and Supplementary Table 2). All mutants contained 3-base substitutions that were determined by the IB or IB-mix used, and there was an accumulation of mutations with repeated rounds of TDEM.

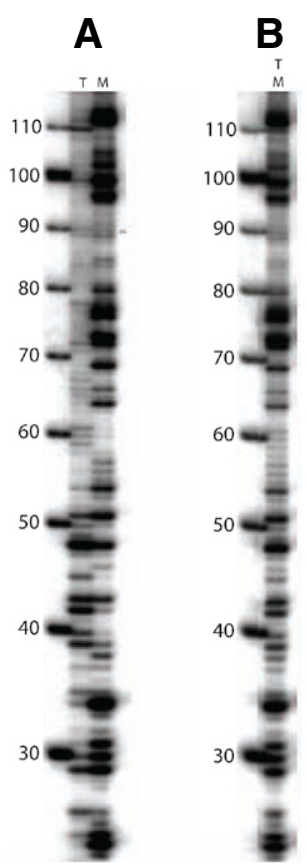

Figure 3. Analysis of target site preference of Mu and Tn7 transposons in lacZa. The sites attacked by the Mu and Tn7 transposons were analyzed as described in the "Materials and methods" section. The left lane represents a10-bp DNA marker, M represents the sites attacked by the Mu transposase, and T represents the sites attacked by the Tn7 transposase. The size and the intensity of labeled fragments indicate the position and the frequency of transposition integration, respectively. (A) The target site preference was analyzed using the $\mathrm{Mu}$ and Tn7 transposition system separately. (B) The reaction mixtures following Mu and Tn7 transposition were mixed and analyzed.

Comparison with epPCR

To determine if a different spectrum of revertants could be obtained using a different mutagenesis method, the lac $Z \alpha$ mutant N19P was subjected to mutagenesis using epPCR, cloned into pCompactKana, and screened on LB agar plates containing $\mathrm{X}$-gal. Of the 856 colonies obtained, we observed only 5 dark blue colonies. Although the 5 clones carried multiple point mutations of 3-6 bases which changed 2 or 3 amino acids, all had a mutation at position 19 that substituted proline with histidine, arginine, leucine, serine, or alanine (Figure 4 and Supplementary Table 2). All of these amino acid substitutions, except for the leucine substitution, had been observed following TDEM with the MI-mix. Furthermore, TDEM performed with MI-mix resulted in a higher reversion frequency than epPCR (Supplementary Table 2).

Mutagenesis of $c c d B$ using TDEM with or without frame-checking procedure We also carried out TDEM of $c c d B$ (38), which exerts a cytotoxic effect on $E$. coli. 


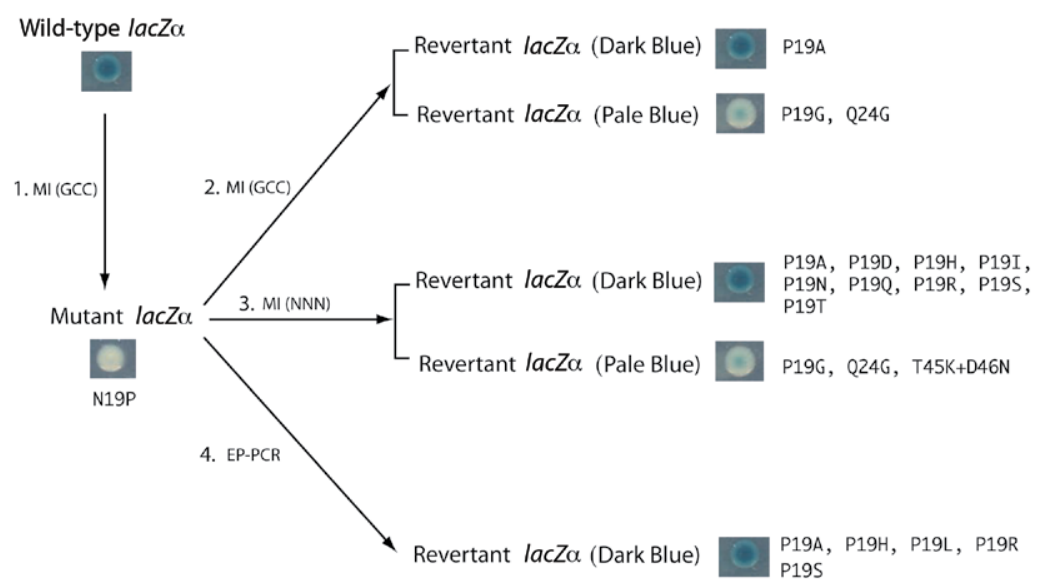

Figure 4. lacZa mutagenesis using TDEM with GCC-MI or MI-mix compared with error-prone PCR (epPCR). Wild-type lacZa was mutated using TDEM with GCC-MI and mutant lacZa lacking a-complementation activity was selected on LB agar plates containing X-gal. Mutant lacZa N19P produces an inactive polypeptide (1). This mutant was subjected to a second round of mutation using various methods: (2) TDEM with GCC-MI, (3) TDEM with MI-mix and (4) epPCR. lacZa from the dark blue colonies or pale blue colonies recovered were sequenced. All revertants, irrespective of the mutagenesis method, showing the dark blue phenotype on X-gal plates had a mutation that replaced P19. However, pale blue colonies having mutations at a distant site were obtained: Q24G and T45K+D46N (3). The revertants recovered from epPCR contained several other point mutations as well as mutations at P19 complicating the interpretation (4). The nucleotide sequences substituted and the frequency of the mutations are shown in Supplementary Table 2.

In this experiment, we characterized the mutants that survived both with and without the frame-checking procedure. To minimize the expression of the $c c d B$ during TDEM, the E. coli lactose promoter (Plac) was not present in pCompact-Kana-CcdB so that after TDEM mutant $c c d B$ needs to be sub-cloned into pFinalScreen, which contains Plac promoter (Supplementary Figure 1). In these experiments, N-terminally deleted $\mathrm{MuA}$ and buffers optimized for increasing the transposition activity and diversity of the integration sites were used because commercial $\mathrm{Tn} 7$ and $\mathrm{Mu}$ transposase showed lot-to-lot variations in the activity (32). After transposition (see Methods) into $c c d B$, we obtained more than 20,000 colonies. When the integration sites were analyzed, they were found to be distributed throughout the $c c d B$ gene, although the possibility of some site preference cannot be excluded (Supplementary Figure 4). This mixture was then analyzed both with and without framechecking.

After performing TDEM with MI-mix without frame-checking, the mutated $\mathrm{CcdB}$ was cloned directly into pFinalScreen, transformed into Top10 cells, and screened. Of the 106 colonies picked and sequenced, 38 contained the intended 3-base substitution, 16 contained a 1-base deletion, 18 contained a 2-base deletion, 31 contained a deletion of $>3$ bases which disrupted the reading frame, and 2 contained a base insertion. Among the 38

\section{Give your marketing programs a LIFT!}

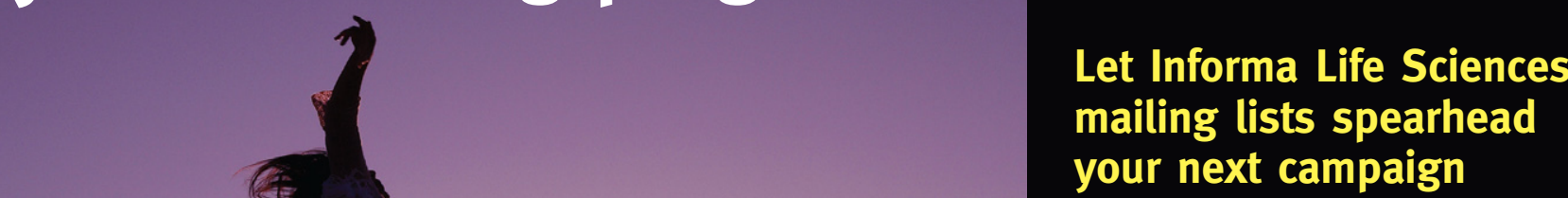

Let Informa Life Sciences your next campaign

If you are looking to maximize your marketing efforts to professionals who work in all aspects of pharma and biotech, you need a resource that gives you access to your best responders - and that's Informa Life Science mailing lists.

Cultivated from our targeted subscriber base of life science professionals, our lists give you the best chance of delivering your brand and offer to this market.

Let us locate the right decision-makers in your market segment:

- Over 125,000 life science professionals

- $\$ 25$ billion in purchasing power

- Segmentation including function, title and laboratory technique

- worldwide reach 
mutants containing a 3-base substitution, 6 contained a stop codon. The remaining 32 colonies contained amino acid substitutions. Thus, only $30 \%$ of clones after TDEM without frame-checking contained the intended 3-base substitution.

In contrast, when TDEM of $c c d B$ was carried out with MI-mix and framechecking, 44 of the 50 clones sequenced had a 3-base substitution (Table 1 ) while 5 had deletions that were multiples of 3 bases so that reading frame was maintained (data not shown). Only one clone had a 1-base deletion mutation. Thus, when the framechecking step is used, the frequency of colonies containing the desired amino acid substitution is dramatically increased.

The amino acid substitution mutants isolated from TDEM without framechecking can be divided into three groups based on the site of mutation: amino acids 2-3, 18-23, and 98 (Table 1). Although most mutations identified following TDEM with frame-checking were located at those 3 positions, novel amino acid substitutions, R31G and L83P+S84C, were also identified (Table 1).

\section{Discussion}

In the current study, we describe a novel mutagenesis method, TDEM, with properties that should facilitate both amino acid scanning mutagenesis and the directed evolution of proteins. TDEM uses $\mathrm{Mu}$ transposase's random integration property and following TDEM of lac $Z \alpha$ and $c c d B$, all mutants that showed phenotypic changes had alterations only at a single site facilitating the interpretation of the effects of different mutants. Furthermore, we showed that repeated rounds of TDEM can be used to accumulate mutations in the target gene. In order to further assess the diversity of the resulting libraries, we performed TDEM without framechecking on the HIV protease gene (300 bp) using MI-mix and randomly sequenced 170 clones from a library of 2 $\times 10^{6}$. All 170 clones had modified bases in the HIV protease gene and 73 clones (43\%) showed the desired 3-base substitution. Among these 73 mutants there were 60 unique HIV protease variants (Supplementary Table 3). Based on these results, we conclude that a single round of TDEM generates libraries with diverse mutations at a single site, although there can be target site preferences depending on the base composition (39).

While we were preparing this manuscript, another group described a similar approach (TriNEx) using a variant $\mathrm{Mu}$ transposon (Mu-Del) containing MlyI sites to substitute three random
A
(1) Wild type

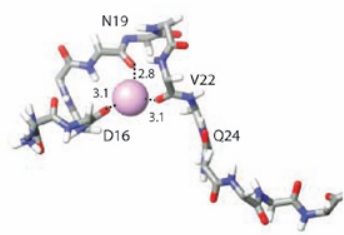

B

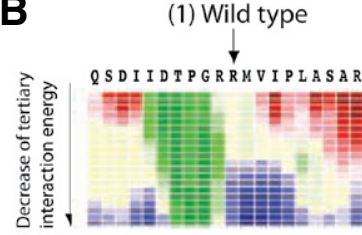

(2) N19P

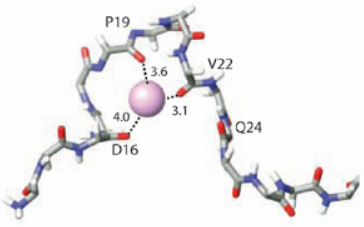

(2) R31A

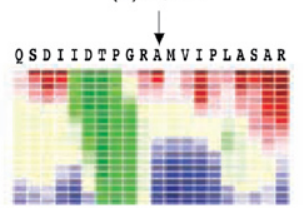

(3) $N 19 P+Q 24 G$

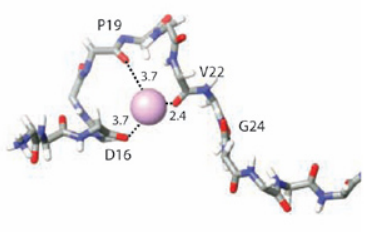

(3) R31G

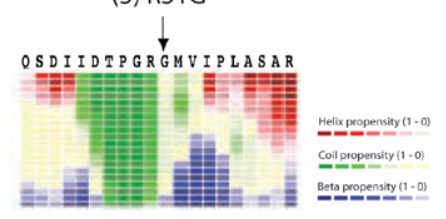

Figure 5. Structural analysis of $\operatorname{lacZa}(A)$ and $\boldsymbol{c c d B}(B)$ mutants. (A) Models of amino acid D16, N19 and V22 of wild-type and mutant lacZa. PDB entry of $E$. coli $\beta$-galactosidase was $1 \mathrm{bpO}$. The wildtype and variants with an all-atom model were energy-minimized using MacroModel (Schrödinger, Portland, ME, USA) with the truncated-Newton conjugated gradient (TNCG) minimization algorithm in generalized born surface area (GBSA) implicit solvation model. The substructure was specified by residues $14-55$, corresponding to the a-peptide (a-donor) and by residues of the $\alpha$-acceptor within $5.0 \AA$ of the a-peptide. The coordinates of the rest of the protein were fixed. The energy-minimized structures of (1) wild-type, (2) N19P, and (3) Q24G variant with N19P of $\beta$-galactosidase are shown with the magnesium ion drawn as a sphere and the main chain of a-peptide shown in stick representation. Distances $(\AA)$ measured between magnesium ion and main chain oxygen of the residues. (B) Change of secondary structure propensity in $c c d B$ mutants; (1) wild-type, (2) R31A and (3) R31G. Three-state secondary structure propensity was calculated using the CSSP2 (http://cssp2. sookmyung.ac.kr) algorithm.

bases for three preexisting bases (40). Using this system, they efficiently isolated $\beta$-lactamase mutants showing increased activity. Because TriNex requires only one MlyI digestion and religation for substituting the three bases, TriNex provides a simple and straightforward approach for generating mutant libraries using transposition. However, this method is limited in that only a maximum of 3 bases can be substituted. Using TriNEX, undesired mutations containing a frameshift, a stop codon, or a changed start codon were seen in 6 out of 49 clones (12\%).

In contrast to TriNEx, TDEM is not limited to single amino-acid substitution, but it can be used to substitute up to 11 bases within the target genes. Although a single amino acid substitution would be sufficient in many experiments, TDEM provides a valuable alternative for generating mutant libraries introducing more than one consecutive amino acid substitution. TDEM has two additional steps compared with TriNEx, and introduces significant numbers of frameshift mutations that are eliminated in the frame-checking step. Importantly, after the frame-checking step, only $2 \%$ of the library contained frameshifts or stop codons, which is less than the percentage of unwanted mutants found in the TriNEx library (however, without the frame-checking procedure, TDEM generates more frameshift mutations than does TriNEx).
Because carbenicillin and ampicillin are biostatic antibiotics and $\beta$-lactamase has robust activity toward these antibiotics, we assumed that $E$. coli containing only a few molecules of $\beta$-lactamase in the periplasm could survive and proliferate. However, we cannot exclude the possibility that the frame-checking step could introduce bias in the mutant library due to sequence preference for exporting the fusion protein. Future efforts will be directed at improving the frame-checking procedure with the goal of minimizing the production of frameshift mutants and simplifying the procedure.

We have used TDEM for the mutagenesis of two genes, $l a c Z \alpha$ and $c c d B$. For lac $Z \alpha$, we first isolated the inactive mutant, N19P. We sequenced only 10 white colonies because the purpose of this initial screen was to isolate a single mutant for generating revertants. We speculated that this N19 mutant did not represent a hot spot of $\mathrm{Mu}$ transposase based on the transposition site analysis (Figure 2) that showed even integration of the Mu transposon throughout lac $Z \alpha$. lac $Z \alpha$ with N19P was then subjected to a second round of TDEM. All revertants showing full activity had a substitution of P19. Several revertants also showed partial activity. Among the mutants showing partial activity was a second site revertant, Q24G. Examination of the crystal structure of $\beta$-galactosidase provided some insights into why $\operatorname{lac} Z \alpha$ 
(N19P) is inactive and why activity is regained in lac $\alpha \alpha(\mathrm{N} 19 \mathrm{P}+\mathrm{Q} 24 \mathrm{G})$ (Figure 5A). Amino acids D16, N19, and V22 coordinate a $\mathrm{Mg}^{2+}$ ion required for enzyme activity $(41,42)$. Comparison of the energyminimized structure of wild-type and N19P showed that in N19P, the distance between the $\mathrm{Mg}^{2+}$ ion and the main chain oxygen atoms interacting with it increases. However, when the Q24G mutation is additionally introduced, there is a significant decrease in the $\mathrm{Mg}^{2+} / \mathrm{D} 16$ and $\mathrm{Mg}^{2+} / \mathrm{V} 22$ distances (Figure 5A). These decreased distances undoubtedly result in more efficient coordination of the $\mathrm{Mg}^{2+}$ and are probably responsible for the more favorable $\alpha$-complementation seen with this second site revertant.

Overall, our results with $c c d B$ mutagenesis were consistent with a previous study in which every residue of CcdB had been individually changed to either Ala, Asp, Glu, Lys, or Arg (43). However, we also identified the $c c d B$ mutants V20Q, Q21W, Q21P, R31G, F98N, F98G, F98Q, and mutants containing 2 amino acid changes that had not been reported previously (Table 1). It was not expected that the mutant R31G would render $c c d B$ inactive since in the previous study (43), the R31 A mutant was active. $\mathrm{R} 31$ is located in the core $\beta$-sheet of the $\mathrm{CcdB}$ monomer structure and mutation of this residue may change the folding stability of the protein. Indeed, when the secondary structure propensity of this region of wild-type and the R31A and R31G mutants were compared (Figure 5B), it was observed that the R31G mutation significantly changed the $\beta$-sheet propensity in the region. In contrast, the R $31 \mathrm{~A}$ mutation does not show any significant change in the secondary structure propensity (Figure $5 \mathrm{~B})$. Thus this analysis suggests that the R31G mutation disrupts the formation of the core $\beta$-sheet that plays a critical role in folding and maintaining the global protein structure.

We have demonstrated that TDEM can be used to introduce alterations at a single position during each round of mutagenesis so that the number of mutations and the sequence to be introduced can be controlled. It is a versatile method in which up to eleven bases within the target can be substituted with any desired sequence. Frame-checking is especially useful for eliminating nonsense mutations when screening for loss-of-function variants. Therefore, TDEM should provide a useful tool for the directed evolution or affinity maturation of a protein as well as for scanning mutagenesis with alanine, cysteine or nonnatural amino acids $(36,37,44,45)$. It should be partic- ularly useful when proteins need to be mutated stepwise to gradually achieve the desired phenotype. TDEM with its versatility, flexibility and controllability facilitates efficient construction of diverse mutant libraries and provides a powerful mutagenesis system.

\section{Acknowled gments}

Y.C.K. conceived the study, designed the experiments and performed the mutagenesis and screening. S.L.M. conceived the study and supervised the work. H.S.L. and S.Y. performed the protein structure analysis of the mutants.

This work was supported in part by a seed grant from the UCLA AIDS Institute [National Institutes of Health (NIH) grant no. AI28697] and by grant no. AI29470 from the NIH. We would like to thank Hans Lee, James Bowie, and Samson Chow for their helpful suggestions. The structure analysis study was supported by the SRC/ERC program of MOST/KOSEF (R11-2005-017-01003-0) and by the Korea Research Foundation Grant funded by the Korean Government (MOEHRD) (KRF-2006-311-C00582). This paper is subject to the NIH Public Access Policy.

The authors declare no competing interests.

\section{References}

1. Clarke, N.D., D.C. Lien, and P. Schimmel. 1988. Evidence from cassette mutagenesis for a structure-function motif in a protein of unknown structure. Science 240:521-523.

2.Zhang, Z., J. Akutsu, M. Tsujimura, and Y. Kawarabayasi. 2007. Increasing in archaeal GlcNAc-1-P uridyltransferase activity by targeted mutagenesis while retaining its extreme thermostability. J. Biochem. 141:553-562.

3. Bridges, B.A. 1969. Mechanisms of radiation mutagenesis in cellular and subcellular systems. Annu. Rev. Nucl. Sci. 19:139-178.

4. Witkin, E.M. 1976. Ultraviolet mutagenesis and inducible DNA repair in Escherichia coli. Bacteriol. Rev. 40:869-907.

5. Mattern, I.E., J.M. van Noort, P. van den Berg, D.B. Archer, I.N. Roberts, and C.A. van den Hondel. 1992. Isolation and characterization of mutants of Aspergillus niger deficient in extracellular proteases. Mol. Gen. Genet. 234:332-336.

6. Kano, H., S. Taguchi, and H. Momose. 1997. Cold adaptation of a mesophilic serine protease, subtilisin, by in vitro random mutagenesis. Appl. Microbiol. Biotechnol. 47:46-51.

7. Kadonaga,J.T. and J.R. Knowles. 1985. A simple and efficient method for chemical mutagenesis of DNA. Nucleic Acids Res. 13:1733-1745.

8. Hutchison, C.A., 3rd, S. Phillips, M.H. Edgell, S. Gillam, P. Jahnke, and M. Smith. 1978. Mutagenesis at a specific position in a DNA sequence. J. Biol. Chem. 253:6551-6560.

9.Sen, S., V. Venkata Dasu, and B. Mandal. 2007. Developments in directed evolution for improving enzyme functions. Appl. Biochem. Biotechnol. 143:212-223.
10. Ho, S.N., H.D. Hunt, R.M. Horton, J.K. Pullen, and L.R. Pease. 1989. Site-directed mutagenesis by overlap extension using the polymerase chain reaction. Gene 77:51-59.

11.Sarkar, G. and S.S. Sommer. 1990. The "megaprimer" method of site-directed mutagenesis. BioTechniques 8:404-407.

12. Cunningham, B.C. and J.A. Wells. 1989. High-resolution epitope mapping of hGH-receptor interactions by alanine-scanning mutagenesis. Science 244:1081-1085.

13. Bass, S.H., M.G. Mulkerrin, and J.A. Wells. 1991. A systematic mutational analysis of hormone-binding determinants in the human growth hormone receptor. Proc. Natl. Acad. Sci. USA 88:4498-4502.

14. Childers, B.M., G.G. Weber, M.G. Prouty, M.M. Castaneda, F. Peng, and K.E. Klose. 2007. Identification of residues critical for the function of the Vibrio cholerae virulence regulator ToxT by scanning alanine mutagenesis. J. Mol. Biol. 367:1413-1430.

15.Sorensen, H., L. Whittaker, J. Hinrichsen, A. Groth, and J. Whittaker. 2004. Mapping of the insulin-like growth factor II binding site of the Type I insulin-like growth factor receptor by alanine scanning mutagenesis. FEBS Lett. 565:19-22.

16. Miyamoto, M., G.D. Han, T. Kimura, Y. Furuichi, and T. Komiyama. 2005. Alaninescanning mutagenesis of HM-1 killer toxin and the essential residues for killing activity. J. Biochem. 137:517-522.

17. Sahin-Toth, M. and H.R. Kaback. 1993. Cysteine scanning mutagenesis of putative transmembrane helices IX and $\mathrm{X}$ in the lactose permease of Escherichia coli. Protein Sci. 2:1024-1033.

18. Frillingos, S., M. Sahin-Toth, J. Wu, and H.R. Kaback. 1998. Cys-scanning mutagenesis: a novel approach to structure function relationships in polytopic membrane proteins. FASEB J. 12:1281-1299.

19. Kuwabara, N., H. Inoue, Y. Tsuboi, N. Nakamura, and H. Kanazawa. 2004. The fourth transmembrane domain of the Helicobacter pylori $\mathrm{Na}+\mathrm{H}+$ antiporter $\mathrm{NhaA}$ faces a water-filled channel required for ion transport. J. Biol. Chem. 279:40567-40575.

20.Low, N.M., P.H. Holliger, and G. Winter. 1996. Mimicking somatic hypermutation: affinity maturation of antibodies displayed on bacteriophage using a bacterial mutator strain. J. Mol. Biol. 260:359-368.

21. Boder, E.T. and K.D. Wittrup. 1997. Yeast surface display for screening combinatorial polypeptide libraries. Nat. Biotechnol. 15:553-557.

22.Liao, H., T. McKenzie, and R. Hageman. 1986. Isolation of a thermostable enzyme variant by cloning and selection in a thermophile. Proc. Natl. Acad. Sci. USA 83:576-580.

23. Bornscheuer, U.T., J. Altenbuchner, and H.H. Meyer. 1998. Directed evolution of an esterase for the stereoselective resolution of a key intermediate in the synthesis of epothilones. Biotechnol. Bioeng. 58:554-559.

24.Stemmer, W.P. 1994. Rapid evolution of a protein in vitro by DNA shuffling. Nature 370:389-391

25. Cadwell, R.C. and G.F. Joyce. 1992. Randomization of genes by PCR mutagenesis. PCR Methods Appl. 2:28-33.

26.Sheryl, B. Rubin-Pitel, C.M.-H.C., Wilfred Chen, Huimin Zhao. 2006. Directed 
evolution tools in bioproduct and bioprocess development, p. 49-72. In S.-T. Yang (Ed.) Bioprocessing for Value-Added Products from Renewable Resources: New Technologies and Applications, Elsevier Science, New York.

27. Murakami, H., T. Hohsaka, and M. Sisido. 2002. Random insertion and deletion of arbitrary number of bases for codon-based random mutation of DNAs. Nat. Biotechnol. 20:76-81

28. Poussu, E., M. Vihinen, L. Paulin, and H. Savilahti. 2004. Probing the alphacomplementing domain of $E$. coli beta-galactosidase with use of an insertional pentapeptide mutagenesis strategy based on $\mathrm{Mu}$ in vitro DNA transposition. Proteins 54:681-691.

29. Anton, B.P. and E.A. Raleigh. 2004 Transposon-mediated linker insertion scanning mutagenesis of the Escherichia coli McrA endonuclease. J. Bacteriol. 186:5699-5707.
30. Haapa, S., S. Taira, E. Heikkinen, and H. Savilahti. 1999. An efficient and accurate integration of mini-Mu transposons in vitro: a general methodology for functional genetic analysis and molecular biology applications. Nucleic Acids Res. 27:2777-2784

31. Biery, M.C., F.J. Stewart, A.E. Stellwagen, E.A. Raleigh, and N.L. Craig. 2000. A simple in vitro Tn7-based transposition system with low target site selectivity for genome and gene analysis. Nucleic Acids Res. 28:1067-1077.

32.Kim, Y.C. and S.L. Morrison. 2008. $\mathrm{N}$-terminal domain-deleted $\mathrm{Mu}$ transposase exhibits increased transposition activity with low target site preference in modified buffers. J. Mol. Microbiol. Biotechnol. Nov 25. [Epub ahead of print]

33. Craig, N.L. 1995. Unity in transposition reactions. Science 270:253-254.

34. Wong, T.S., K.L. Tee, B. Hauer, and U. Schwaneberg. 2004. Sequence saturation

\section{HOT AND COLD DRY BATHS}

\section{Cycle From $-40^{\circ} \mathrm{C}$ to $+100^{\circ} \mathrm{C}$}

\author{
Designed for lab and \\ research facilities the CPV \\ family of cold plates \\ delivers a temperature \\ controlled, programmable \\ dry bath. They are used to \\ maintain sample \\ temperature or to cycle \\ between multiple \\ temperatures.
}

\section{Standard Features}

- Data logging

- Ramp and soak programming

- Internal and external RTD sensor

- Quiet fan design

- Front panel or computer control

- Flat surface: plain or tapped

- $0.1^{\circ} \mathrm{C}$ resolution

\section{Standard Options}

- Acrylic cover

- Wide temperature range cascades

- Cold well

Visit www.teca.us for more versatile cooling products for lab and industry

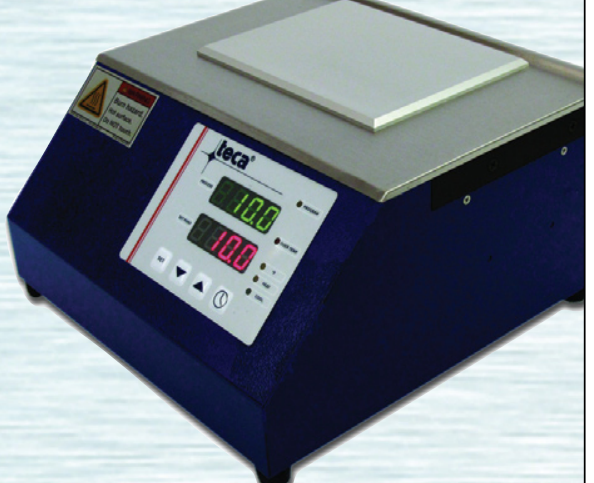

Standard Versions

- AHP-1200CPV 13.3" X 5.8" plate

- AHP-301CPV 6.0 " X 4.5" plate

- AHP-1200DCP (dual plate)

- AHP-1200CPV for cascade option

- AHP-800MSP stirring cold plate

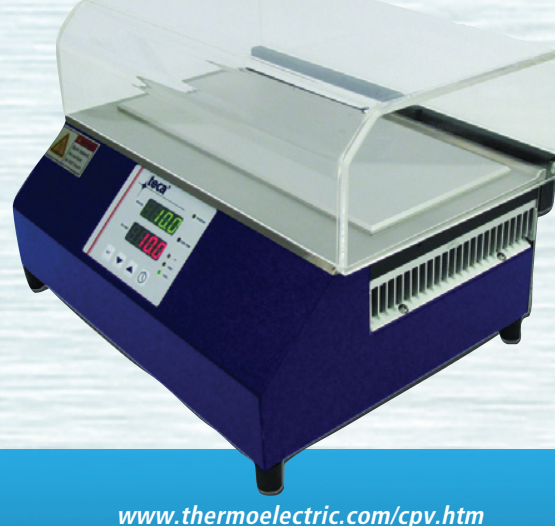

Www. thermoelectric.com/cpv.htm

Contact TECA today! 888-832-2872 mutagenesis (SeSaM): a novel method for directed evolution. Nucleic Acids Res. 32:e26.

35. Boeke, J.D. and P. Model. 1982. A prokaryotic membrane anchor sequence: carboxyl terminus of bacteriophage $\mathrm{f} 1$ gene III protein retains it in the membrane. Proc. Natl. Acad. Sci. USA 79:5200-5204.

36. Wang, L. and P.G. Schultz. 2001. A general approach for the generation of orthogonal tRNAs. Chem. Biol. 8:883-890.

37. Wang L, T.J. Magliery, D.R. Liu, and P.G. Schultz. 2000. A new functional suppressor tRNA/aminoacyl-tRNA synthetase pai for the in vivo incorporation of unnatural amino acids into proteins. J. Am. Chem. Soc. 122:5010-5011.

38. Bernard, P. and M. Couturier. 1992 Cell killing by the $\mathrm{F}$ plasmid $\mathrm{CcdB}$ protein involves poisoning of DNA-topoisomerase II complexes. J. Mol. Biol. 226:735-745.

39. Haapa-Paananen, S., H. Rita, and H. Savilahti. 2002. DNA transposition of bacteriophage $\mathrm{Mu}$. A quantitative analysis of target site selection in vitro. J. Biol. Chem. 277:2843-2851.

40.Baldwin, A.J., K. Busse, A.M. Simm, and D.D. Jones. 2008. Expanded molecular diversity generation during directed evolution by trinucleotide exchange (TriNEx). Nucleic Acids Res. 36:e77.

41.Juers, D.H., R.H. Jacobson, D. Wigley, X.J. Zhang, R.E. Huber, D.E. Tronrud, and B.W. Matthews. 2000. High resolution refinement of beta-galactosidase in a new crystal form reveals multiple metal-binding sites and provides a structural basis for alphacomplementation. Protein Sci. 9:1685-1699.

42.Gallagher, C.N. and R.E. Huber. 1999. Stabilities of uncomplemented and complemented M15 beta-galactosidase (Escherichia coli) and the relationship to alpha-complementation. Biochem. Cell Biol. 77:109-118

43. Bajaj, K., P. Chakrabarti, and $R$. Varadarajan. 2005. Mutagenesis-based definitions and probes of residue burial in proteins. Proc. Natl. Acad. Sci. USA 102:16221-16226.

44.Noren, C.J., S.J. Anthony-Cahill, M.C. Griffith, and P.G. Schultz. 1989. A general method for site-specific incorporation of unnatural amino acids into proteins. Science 244:182-188

45.Bain, J.D., E.S. Diala, C.G. Glabe, D.A. Wacker, M.H. Lyttle, T.A.Dix, and A.R. Chamberlin. 1991. Site-specific incorporation of nonnatural residues during in vitro protein biosynthesis with semisynthetic aminoacyltRNAs. Biochemistry 30:5411-5421.

Received 22 October 2008; accepted 23 March 2009.

Address correspondence to Yun Cheol Kim, Department of Microbiology, Immunology and Molecular Genetics, David Geffern School of Medicine, University of California Los Angeles 405 Hilgard Avenue, Los Angeles, CA, 90095. USA.email: kyc8402@ucla.edu 